\title{
SR-MAC \\ Um Protocolo Cross-Layer baseado em Coloração de Grafos para Melhoria da Eficiência de Redes de Sensores Sem Fio
}

\author{
Bruno Rogério S. dos Santos ${ }^{1}$, Leonardo S. Rocha ${ }^{1}$, \\ Joaquim C. Júnior ${ }^{1}$, Renan da S. Alves ${ }^{1}$ \\ ${ }^{1}$ Mestrado Acadêmico em Ciência da Computação (MACC) \\ Universidade Estadual do Ceará (UECE) \\ Caixa Postal 1531 - 60.714.903 - Fortaleza - CE - Brazil \\ brunoro1389@gmail.com, lsampaiorocha@gmail.com \\ celestinodlarces.uece.br, renan.alvesdaluno.uece.br
}

\begin{abstract}
In this paper is presented the SR-MAC protocol, a cross-layer protocol based on the graph coloring for wireless sensor networks. The main characteristic of the protocol is an efficient use of the time slots allocated to the sensors, through a TDMA scheduling, offering scalability and efficiency in the transmission of collected data to the base station. The SR-MAC was validated through a specific simulator for sensor networks. The protocol presented good results when compared to protocols that do not reuse time slots, even in high density scenarios, being able to support $53 \%$ more nodes and having an energy efficiency in the data collection superior in more than $30 \%$.
\end{abstract}

Resumo. Neste artigo é apresentado o protocolo SR-MAC, um protocolo crosslayer baseado em coloração de grafos para redes de sensores sem fio. A principal característica do protocolo é um uso eficiente dos intervalos de transmissão alocados aos sensores, por meio de um escalonamento TDMA, oferecendo escalabilidade e eficiência na transmissão dos dados coletados para a estação base. O SR-MAC foi validado por meio de um simulador específico para redes de sensores. O protocolo apresentou bons resultados quando comparado a protocolos que não fazem reuso dos intervalos de transmissão, mesmo em cenários com alta densidade, sendo capaz de suportar 53\% mais nós e possuindo uma eficiência energética na coleta de dados superior em mais de $30 \%$.

\section{Introdução}

Uma rede de sensores sem fio (RSSF) consiste em sensores autônomos distribuídos espacialmente para monitorar condições físicas ou ambientais, tais como temperatura, som, vibração, pressão, movimento ou poluentes e passar cooperativamente seus dados através da rede para um local principal [Goyal and Tripathy 2012], responsável por processar tais dados e repassar a um usuário final. Este local é comumente conhecido como estação base. Por apresentarem características de comunicação sem fio, estes dispositivos precisam compartilhar o meio para uma transmissão de dados eficiente. Na necessidade de coordenar transmissões em ambientes deste tipo, a fim de que interferências e colisões sejam evitadas, são necessários protocolos para regulamentar o acesso ao meio [Huang et al. 2013]. 
Através do escalonamento TDMA, são atribuídos intervalos de tempo a cada sensor, de modo que cada sensor tenha seu determinado momento para transmitir seus dados, não interferindo na transmissão de outro sensor [Sgora et al. 2015]. Isso possibilita que a estação base faça um escalonamento da rede atribuindo faixas de tempo aos sensores, tomando cuidado com o sincronismo entre todos os componentes da rede. O escalonamento em redes de sensores também pode ser útil na diminuição do consumo de energia das baterias dos nós, pois através do escalonamento é possível fazer com que determinados sensores realizem transmissões e recepções de dados sem que haja colisões, enquanto outros nós ficarão em estado de espera, consequentemente, diminuindo o consumo de energia.

Além dos problemas relacionados à interferência, colisão de dados e o consumo de energia [Cunha et al. 2013], outro aspecto importante que deve se levar em conta em uma rede de sensores, é a escalabilidade da rede e a eficiência energética em relação a quantidade de dados entregues à estação base. Um protocolo escalável permite que uma topologia de rede aceite a entrada de mais nós sem piorar o desempenho e o funcionamento da rede. Quanto a eficiência de coleta, uma rede é eficiente na coleta de dados quando os sensores conseguem entregar uma grande quantidade de dados à estação base levando em conta o consumo de energia de suas baterias.

Neste artigo propõe-se o protocolo SR-MAC (Spatial Reuse-Medium Access Control), cuja principal característica é a reutilização dos intervalos de tempo de um quadro TDMA. O reuso dos intervalos tem como finalidade tornar o protocolo escalável, maximizar o desempenho da rede e as trocas de informações e tornar o protocolo eficiente em relação à coleta de dados da estação base. Para isso foi utilizada a heurística de coloração de grafos [Rocha 2012].

O restante do artigo está organizado da seguinte forma: a seção 2 aborda os trabalhos relacionados. A seção 3 apresenta o protocolo SR-MAC e suas características. Na seção 4 apresenta-se a configuração do ambiente experimental e os resultados obtidos, enquanto a seção 5 conclui o artigo e apresenta perspectivas de trabalhos futuros.

\section{Trabalhos Relacionados}

A fim de possibilitar escabilidade, eficiência na coleta de dados pela estação base e evitar o desperdício de tempo, no intuito de maximizar o desempenho e as trocas de informações em torno da rede é que foi aplicada a ideia da reutilização dos intervalos de tempo através de um escalonamento TDMA no protocolo SR-MAC. Além disso, protocolo SR-MAC também se preocupa com o atraso das transmissões por ser um protocolo aplicado para rede de sensores de múltiplos saltos [El Gamal and Kim 2011]. Os protocolos a seguir foram escolhidos para comparação pela semelhança com o protocolo proposto.

O Protocolo SOTP [Wang et al. 2006] é um protocolo cross-layer baseado no esquema de acesso ao meio TDMA [Mitra 2009]. O SOTP adota um escalonamento inverso como forma de reduzir eficientemente os atrasos das transmissões em uma rede de múltiplos saltos. Esse escalonamento consiste na alocação de intervalos de tempo do fim ao início do quadro TDMA aos sensores da rede. Assim serão alocados aos primeiros nós que se registrarem na rede, os intervalos de transmissão dispostos no fim do quadro e aos últimos nós a se registrarem, os intervalos dispostos no começo do quadro. Este processo ocorre até que todos os nós da rede estejam registrados. Desta forma, a 
informação originada em um nó poderá ser entregue à estação base em um único quadro, diminuindo-se assim os atrasos. A diferença do SOTP para o protocolo proposto se dá pela não reutilização dos intervalos de transmissão.

No que se refere ao reuso dos intervalos TX, Gajjar et al. [Gajjar et al. 2014] propuseram um protocolo, flexível, eficiente em energia e de baixa latência, chamado SOFLEE (do inglês, Self Organized, Flexible, Latency and Energy Efficient). Neste protocolo a alocação dos intervalos de tempo é feita centralmente pela estação base. A alocação do mesmo intervalo TX acontece para os nós que estão há dois saltos ou níveis de distância. A rede no SOFLEE é setorizada em níveis, nós que estão distantes a dois níveis podem compartilhar o mesmo intervalo TX, proporcionando aumento na reutilização espacial do canal e diminuição na latência de dados. Já no SR-MAC a alocação dos intervalos TX também acontece centralmente pela estação base, porém utilizando um algoritmo de coloração que definirá os critérios de reutilização ou não do intervalo de transmissão.

Na mesma direção, porém usando uma heurística de grafos para possibilitar o reuso de intervalos de tempo, Wu et al. [Wu et al. 2010] propuseram um protocolo de escalonamento TDMA distribuído baseado em um algoritmo de coloração, fazendo uso da reutilização espacial do canal de transmissão, ou seja, possibilitando que diferentes sensores utilizem o mesmo canal de transmissão. Porém deve-se levar em consideração alguns aspectos, como o raio de alcance e a potência de transmissão dos sensores. O protocolo TDMA-CA aloca diferentes cores para os nós conflitantes na rede e providencia intervalos de tempo distintos para a transmissão de dados para cada cor através do escalonamento TDMA. O algoritmo de coloração do protocolo TDMA-CA serviu como base para a criação do algoritmo de coloração proposto para o protocolo SR-MAC, porém o algoritmo do TDMA-CA é distribuído, com os nós de rede participando ativamente no processo de coloração, o que difere do algoritmo proposto neste artigo, cuja coloração é de responsabilidade somente da estação base.

\section{O Protocolo SR-MAC}

O protocolo SR-MAC é um protocolo cross-layer que apresenta aspectos relevantes em relação ao roteamento da rede, porém se notabiliza pelo uso eficiente da camada de enlace, precisamente a parte que se refere ao controle de acesso ao meio (MAC). MAC (do inglês, Medium Access Control) é um termo utilizado para designar parte da camada de enlace e que tem como responsabilidade, transferir os pacotes de forma segura de um nó até o próximo nó adjacente, especificando as regras para transmissão dos dados pelo enlace [Kurose and Ross 2010].

O SR-MAC oferece um reuso eficiente dos intervalos por meio de um escalonamento TDMA utilizando a técnica de coloração de grafos. Utiliza mecanismos do SOTP [Wang et al. 2006] acrescentado de mecanismo para reuso de intervalos de transmissão e economia de recursos. O escalonamento é centralizado na estação base e acontece através da aplicação de um algoritmo de coloração. O reuso dos intervalos de transmissão irá potencializar o ciclo de trabalho dos sensores e tornar eficiente a coleta de dados da estação base. Este reaproveitamento tornará a rede escalável.

A coloração de grafos em uma rede de sensores consiste em atribuir cores, as quais estão associadas aos intervalos de tempo disponíveis em um quadro TDMA, aos sensores da rede. A medida que os nós forem se registrando na rede, serão alocados a eles 
estes intervalos, e cada intervalo está associado a uma determinada cor. Para que um nó, ainda não registrado ou que entrou recentemente na rede, possa utilizar um intervalo que já esteja sendo utilizado por outro sensor devem ser satisfeitas algumas condições. Estas condições serão explicadas na subseção 3.2.

O processo de registro realizado pelo protocolo SR-MAC é auto-organizado e semelhante ao protocolo SOTP. A operação no SR-MAC é dividida em quadros, cada quadro dividido em intervalos de tempo de mesma duração. Cada sensor tem seu próprio quadro, este quadro deve está sincronizado com os demais quadros da rede, incluindose o quadro da estação base, a fim de que o processo de registro do SR-MAC funcione perfeitamente.

Um quadro consiste de cinco tipos de intervalo de tempo: intervalo broadcast (BRS), intervalo de detecção de portadora (CSS), intervalo de transmissão (TX), intervalo de recepção (RX) e intervalo ocioso (IDS). Cada quadro possui um intervalo de tempo dedicado à estação base, o intervalo BRS, e um intervalo para detecção de portadora, o intervalo CSS. Os intervalos BRS e CSS são sempre o primeiro e segundo intervalo de tempo de um quadro, respectivamente. Estes intervalos aparecem no começo do quadro para que se tenha um melhor controle da rede e do processo de registro pela estação base. Cada nó passa por três estados: busca, sincronização e registro. Um nó registrado pode ter apenas um pai, porém nada o impede de possuir muitos nós filhos. A seguir é descrito o processo de registro dos sensores junto à estação base no protocolo SR-MAC.

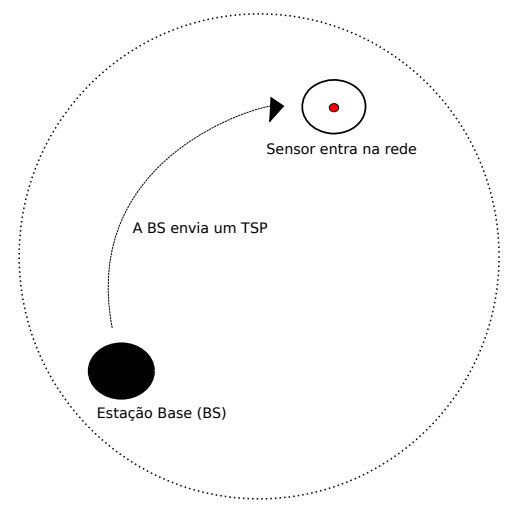

Figura 1. Estado de Busca

No estado de busca, representado pela Figura 1, o sensor escuta transmissões que estão dentro do seu raio de alcance, seja da estação base ou de outros sensores. Nesse estado, o sensor espera receber da estação base um TSP (do inglês, Time Slot Packet), um pacote de atribuição de intervalo de tempo, no qual contêm as informações referentes aos intervalos que estão ocupados e desocupados no quadro TDMA, além das informações referentes ao estado de cada sensor.

O pacote TSP é enviado pela estação base, por meio de transmissão broadcast no intervalo BRS, a todos os sensores ativos na rede, a cada quadro TDMA que se repete. Ao receber o TSP, o sensor move-se para o estado de sincronização. 


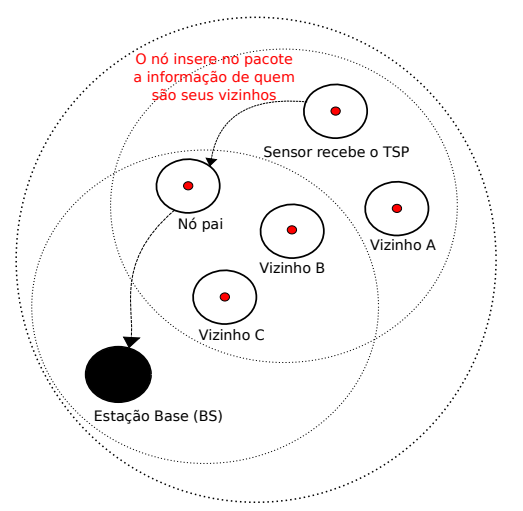

Figura 2. Estado de Sincronização

No estado de sincronização, representado pela Figura 2, o sensor escolhe um intervalo para ser o seu TX, a partir do TSP que recebeu, e um nó vizinho para ser o seu nó pai. Depois adiciona essas informações em um pacote REG que enviará ao nó pai escolhido, além de adicionar também informações sobre os ID's dos sensores vizinhos, no intuito de informar à estação quem são seus vizinhos e assim gerar o grafo de conflitos. O nó envia esse pacote REG tanto no intervalo CSS como no intervalo TX que escolheu. O nó pai por sua vez encaminha o pacote REG para o seu respectivo nó pai, e assim sucessivamente, até o pacote REG chegar à estação base.

Ao chegar na estação base, é aplicado um algoritmo de coloração no grafo de conflitos criado a partir das informações do pacote REG recebido. Este algoritmo será explicado na subseção 3.2 desta seção. O algoritmo é aplicado com base nas informações do nó pai escolhido, dos ID's dos nós vizinhos e dos nós filhos dos seus vizinhos. A partir da coloração do grafo de conflitos é possível saber se irá ser reutilizado ou não um determinado intervalo TX para o nó que quer se registrar.

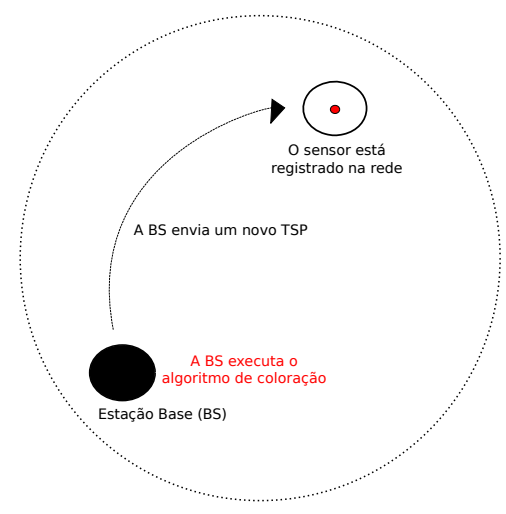

Figura 3. Estado de Registro

Na Figura 3, após alocar o intervalo TX para o nó, por meio do algoritmo de coloração, a estação base atualiza o TSP e o envia a todos os sensores da rede. Isso é necessário para que todos os sensores saibam quais nós atualmente estão registrados na rede e para que os nós pais, caso necessário, modifiquem seus intervalos $\mathrm{RX}$ de acordo com o intervalo TX do nó filho, pois o intervalo TX de um dos seus nós filhos pode ter 
sido alterado ou não pela estação base durante o processo de registro. Ao receber o novo TSP, o sensor sabe se foi registrado ou não e se o intervalo que ele escolheu foi mantido ou não pela estação base. Assim, o sensor ao verificar que está registrado, move-se para o estado de registro e começa a colher dados e enviar para a estação base.

No caso de dois ou mais nós selecionarem o mesmo intervalo TX durante o processo de registro, a estação aloca diferentes intervalos para estes sensores, caso não se respeite as condições de coloração. Só há necessidade de retransmissões neste protocolo, se as informações de registro não chegarem até o destinatário final, no caso a estação base. Depois de todos os nós serem registrados, a árvore de roteamento da rede é terminada, assim é possível saber a rota que cada pacote transmitido pelos sensores irá trafegar. Com a aplicação da heurística de coloração de grafos é possível a ocorrência de múltiplas transmissões simultâneas evitando interferências e conflitos entre as transmissões dos sensores.

\subsection{Roteamento da Rede}

As rotas que interligam um sensor até a estação base são chamadas de caminhos de múltiplos saltos. Por utilizar-se de múltiplos saltos para o tráfego das informações, o protocolo SR-MAC adota a estratégia do escalonamento inverso, relatada na seção 2. Esta estratégia diminui o atraso em transmissões de múltiplos saltos, pois aumenta a probabilidade de que as informações transmitidas por um nó chegue à estação base em um único quadro. Porém quando há muitas reutilizações de intervalos de tempo e um nó pai com vários nós filhos, não há garantia de que a estratégia funcione dessa maneira. De qualquer forma, diminui-se o número de quadros necessários para que as informações cheguem até a estação base.

A fim de obter eficiência na implementação do escalonamento inverso foi criada uma heurística para ordenar a entrada dos nós no processo de registro. Para isso foi dado aos nós localizados mais próximos à estação base, os intervalos de tempo dispostos no final do quadro e aos nós localizados distantes, os intervalos de tempo disponíveis no começo do quadro, após os intervalos de tempo 0 e 1, que são os intervalos BRS e CSS, respectivamente. $\mathrm{O}$ algoritmo abaixo obtém a ordenação dos nós em relação à entrada no processo de registro. Dessa forma, os nós que estão próximos à estação base irão esperar menos tempo pra entrar no processo de registro do que os nós mais distantes da estação base.

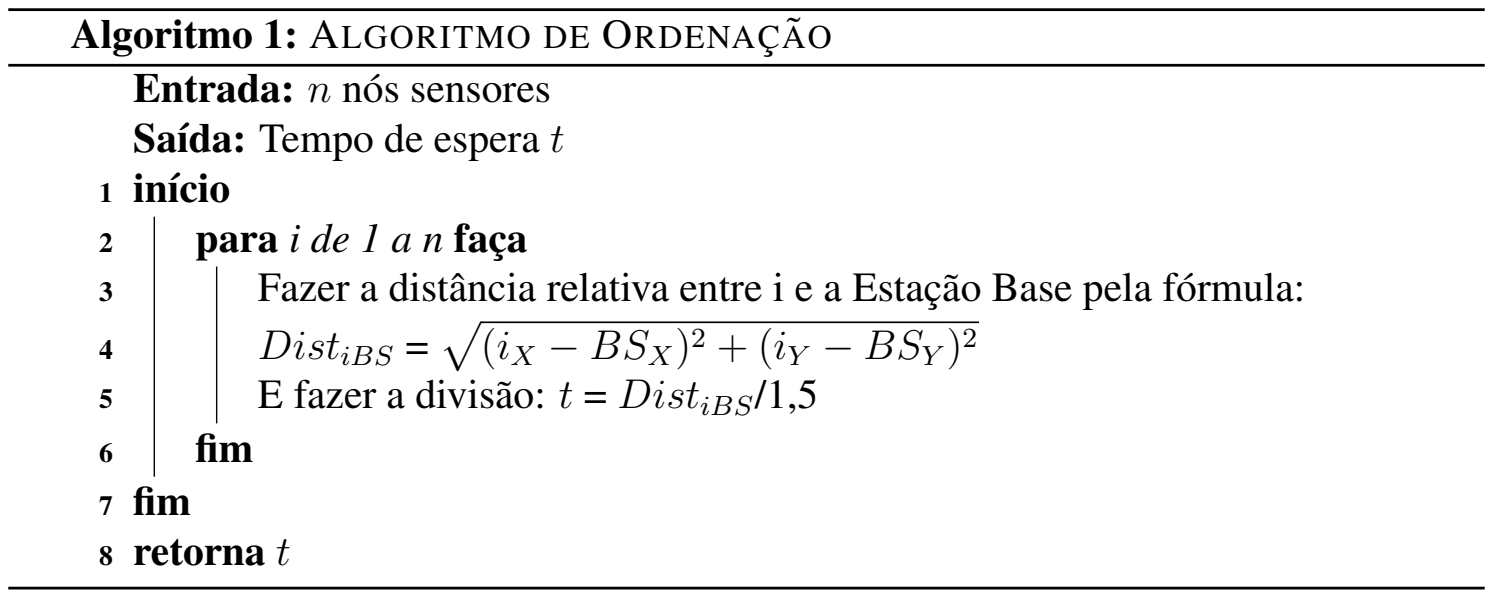


Onde $i$ representa o ID de cada sensor e $n$ o número de nós inseridos da rede. $B S_{X}$ e $B S_{Y}$ representam, respectivamente, as coordenadas $\mathrm{X}$ e $\mathrm{Y}$ da estação base, e $i_{X}$ e $i_{Y}$ representam, respectivamente, as coordenadas $\mathrm{X}$ e Y do sensor $i$. O cálculo da distância relativa entre cada sensor e a estação base tem como resultado o valor da distância em metros entre o sensor e a estação base. Essa distância representada pela variável Dist $_{i B S}$ é dividida por 1,5, valor esse que foi definido empiricamente a partir do comportamento dos nós na rede através de mais de 50 simulações, para cada topologia simulada. $\mathrm{O}$ valor dessa divisão retorna um tempo de espera específico para cada nó, ou seja, o tempo que um nó deve aguardar para entrar no processo de registro sem atrapalhar o processo de registro de outro nó.

Com esse algoritmo evita-se que todos os nós tentem se registrar ao mesmo tempo, o que resultaria em conflitos, e que não precisem esperar tanto tempo para entrar no processo de registro junto à estação base, minimizando o tempo de registro de todos os nós na rede. Nos experimentos mostrados na próxima seção, observou-se que esta estratégia resultou em uma boa organização da rede, evitando o uso de recursos desnecessários e possibilitando uma maior diversidade de nós pais.

Em relação a construção da árvore de roteamento, o critério de escolha do nó pai/filho implementado está relacionado com as distâncias dos nós sensores que querem se registrar com as distâncias dos seus vizinhos registrados e a distância em relação à estação base. A escolha dos nós pais se dá pelas equações 1, 2 e 3. Dessa forma o nó escolhido para ser o nó pai será o sensor que estiver na melhor posição entre o nó não registrado e a estação base quando comparado às distâncias dos outros vizinhos do nó que está no processo de registro, ou seja, será o nó pai o sensor que estiver mais próximo do nó não registrado e da estação base.

A escolha deste critério de nó pai/filho tem como objetivo fornecer estabilidade na comunicação entre os sensores e diminuir o consumo de energia das baterias. Estabilidade por causa do posicionamento do nó pai entre os seus nós filhos e a estação base e, também, economia de energia das baterias dos sensores pelo fato da pouca distância entre o nó filho e o nó pai.

$$
\begin{gathered}
D_{N V}=\sqrt{\left(N_{X}-V_{X}\right)^{2}+\left(N_{Y}-V_{Y}\right)^{2}} \\
D_{V B S}=\sqrt{\left(V_{X}-B S_{X}\right)^{2}+\left(V_{Y}-B S_{Y}\right)^{2}} \\
D_{N B S}=\sqrt{\left(N_{X}-B S_{X}\right)^{2}+\left(N_{Y}-B S_{Y}\right)^{2}}
\end{gathered}
$$

$D_{N V}, D_{V B S}$ e $D_{N B S}$ representam, respectivamente, a distância entre um nó não registrado e cada vizinho registrado, vizinho registrado e a estação base e um nó não registrado e a estação base. $N_{X}$ e $V_{X}$ representam, respectivamente, as coordenadas X de um nó que deseja se registrar e dos vizinhos registrados desse nó. E por último, $N_{Y}$ e $V_{Y}$ representam, respectivamente, as coordenadas Y de um nó não registrado e dos vizinhos registrados desse nó. 


\subsection{Escalonamento da Rede}

O aprimoramento do escalonamento da rede no protocolo SR-MAC em relação a outros protocolos, se deve a implementação da reutilização dos intervalos de transmissão. Para implementar o reuso dos intervalos TX já utilizados por nós registrados na rede, utilizou-se o modelo de coloração de grafos, mais precisamente a coloração de vértices. Primeiramente é criado um grafo de conflitos, este grafo é construído através da matriz de adjacência armazenada na estação base. Esta matriz é criada a partir das informações fornecidas por cada sensor presente na rede, durante o processo de registro.

Com este grafo, a estação base sabe a vizinhança de cada sensor e assim o grafo de conflitos pode ser colorido, respeitando as regras de interferências citadas abaixo. Dessa forma é atribuída uma cor para cada nó da rede, sendo que esta cor é associada a cada intervalo de tempo existente no quadro TDMA, com exceção do intervalo BRS e do intervalo CSS. Para que os nós possam ser coloridos com a mesma cor, ou seja, receberem o mesmo intervalo TX de outros sensores, eles devem satisfazer as seguintes condições:

1) Os nós não podem possuir o mesmo intervalo TX dos seus vizinhos, assim a transmissão de um não interferirá na transmissão do outro, evitando a ocorrência de conflitos, como por exemplo, o problema do terminal exposto.

2) Os nós não podem possuir o mesmo intervalo TX dos vizinhos do nó pai escolhido, assim evitará a ocorrência do problema do terminal oculto em que dois nós que estão fora do alcance um do outro acabam transmitindo ao mesmo tempo para um mesmo nó, pois em redes de sensores um sensor transmite para todos os nós que estão dentro do seu raio de alcance e não somente para o nó destinatário.

3) Os nós não podem possuir o mesmo intervalo TX dos nós filhos de seus vizinhos, assim também evita outra possibilidade de ocorrência do problema do terminal oculto, evitando que ocorra colisão de dados em um dos seus vizinhos quando transmitir para todos os sensores que estão no seu raio de transmissão.

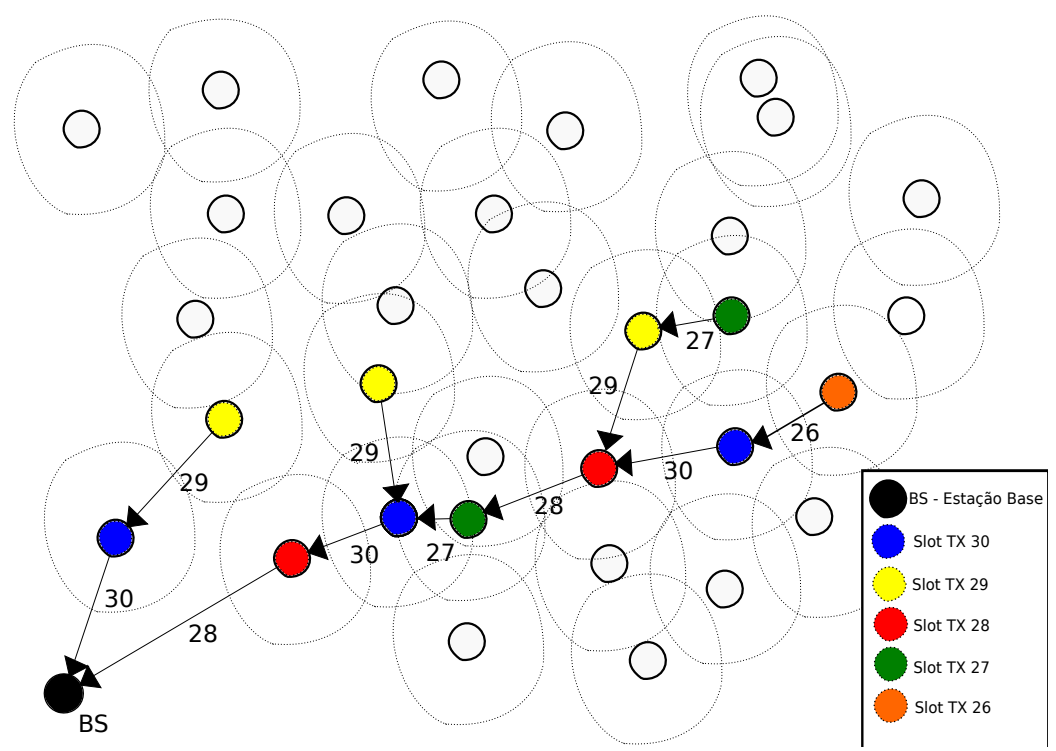

Figura 4. Exemplo de utilização do protocolo SR-MAC em alguns nós sensores da rede. 
A Figura 4 mostra um exemplo de utilização do protocolo SR-MAC em alguns nós da rede. Para um mesmo cenário e a mesma quantidade de nós registrados podemos ver que houve reutilização dos intervalos de transmissão, pois quando a estação base aplicou o algoritmo de coloração nos sensores foi possível reaproveitar os intervalos sem causar interferência e conflito nas transmissões dos outros nós da rede. Assim, neste exemplo com 11 nós registrados foram precisos somente 5 intervalos de transmissão.

O algoritmo que retrata a coloração dos nós sensores a partir dos seus intervalos de transmissão, segue abaixo:

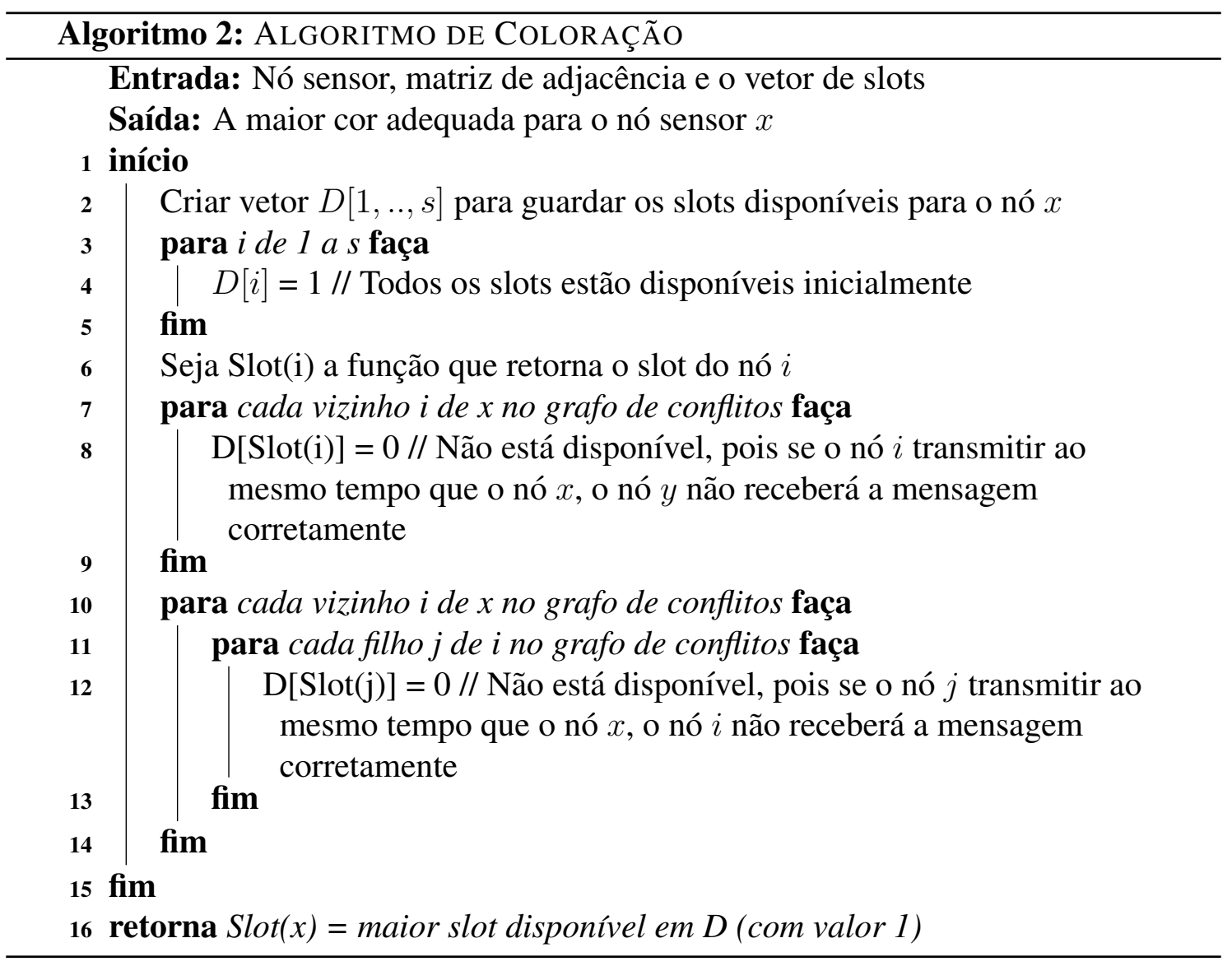

\section{Análise das Simulações}

Para a simulação da rede foi utilizado o Castalia, na versão 3.3 [Boulis et al. 2011]. Este software é um simulador de eventos discretos específicos para redes de sensores sem fio, sendo uma extensão do simulador de redes $\boldsymbol{O M N e T + + , ~ c u j a ~ v e r s a ̃ o ~ u t i l i z a d a ~ f o i ~ a ~} \mathbf{4 . 6}$ [Varga 2014]. O Castalia oferece modelagens realísticas do canal sem fio, gerenciamento da bateria e módulos de rádio de cada nó sensor. Para comparação com os resultados apresentados pelo SR-MAC ${ }^{1}$, mediante as métricas utilizadas, foi simulado também o protocolo SOTP.

Os nós sensores foram depositados no ambiente de forma aleatória seguindo uma distribuição uniforme e não possuindo nenhum aspecto de mobilidade. Cada nó, exceto a

\footnotetext{
${ }^{1}$ Link para download da implementação do SR-MAC no Castalia: https://bitbucket.org/masks/srmac/downloads/
} 
estação base, foi configurado com uma energia inicial de 10 joules para que as simulações não demorassem muito tempo. Foram utilizadas topologias com 50, 100 e 150 nós e uma área de cobertura $100 \mathrm{~m}$ x 100m.

Tabela 1. Cenários utilizados para as simulações dos protocolos.

\begin{tabular}{|c|c|c|c|}
\hline Cenário & Quantidade de Nós & Área $(\mathrm{m} \times \mathrm{m})$ & Posição da Estação Base \\
\hline 1 & 50 & $100 \times 100$ & $(10,10)$ \\
\hline 2 & 100 & $100 \times 100$ & $(10,10)$ \\
\hline 3 & 150 & $100 \times 100$ & $(10,10)$ \\
\hline
\end{tabular}

Os protocolos foram executados em execuções parciais de 200, 400, 600, 800 e 1000 segundos a fim de obter um resultado comparativo para as métricas analisadas em cada um desses períodos. A estação base foi configurada com uma potência muito alta, a fim de que seu raio de transmissão alcance todos os nós da rede, para que assim, os pacotes TSP sejam enviados a todos os sensores, de modo que o processo de registro dos nós na rede tenha êxito. Já os sensores foram configurados com uma potência muito baixa. O valor configurado para a duração de cada intervalo de tempo do quadro foi de 5 milissegundos.

O modelo de interferência acumulativa foi utilizado nas simulações dos protocolos. Os nós foram configurados para transmitir o máximo de pacotes dentro do intervalo de transmissão dedicado a eles. O intuito foi de avaliar o desempenho dos dois protocolos quanto ao número de transmissões por intervalo TX. Sendo assim possível medir a eficiência de pacotes transmitidos pelos sensores durante o mesmo período de tempo.

Os valores para o tamanho do quadro foram configurados de acordo com o número de sensores presentes em cada cenário, mais o intervalo de tempo dedicado a estação base, ou seja o intervalo BRS, levando-se em conta também a duração de cada intervalo de tempo, que é de 5 milissegundos. Por exemplo, para um cenário com 50 nós, o tamanho do quadro será 0,255 segundos, pois (50 intervalos $\mathrm{TX}+1$ intervalo $\mathrm{BRS}) * 5 \mathrm{~ms}=0,255$ segundos.

Dessa forma, o tamanho do quadro na simulação do protocolo SOTP para os cenários 1,2 e 3 foram configurados com os valores de 0,255 segundos, 0,505 segundos e 0,755 segundos, respectivamente, de acordo com o número de sensores presentes em cada cenário e a duração do intervalo de tempo. Já para o protocolo SR-MAC, de acordo com a quantidade de intervalos de transmissão necessária para cada topologia, que diferentemente do protocolo SOTP, não é necessário que a quantidade de intervalos TX seja a mesma que a quantidade de sensores presentes na rede. A quantidade de intervalos TX necessária para cada topologia no SR-MAC, observada através das simulações, é mostrada na Tabela 2. Os cenários 1, 2 e 3 foram configurados para 0,18 segundos, 0,305 segundos e 0,355 segundos, respectivamente. 


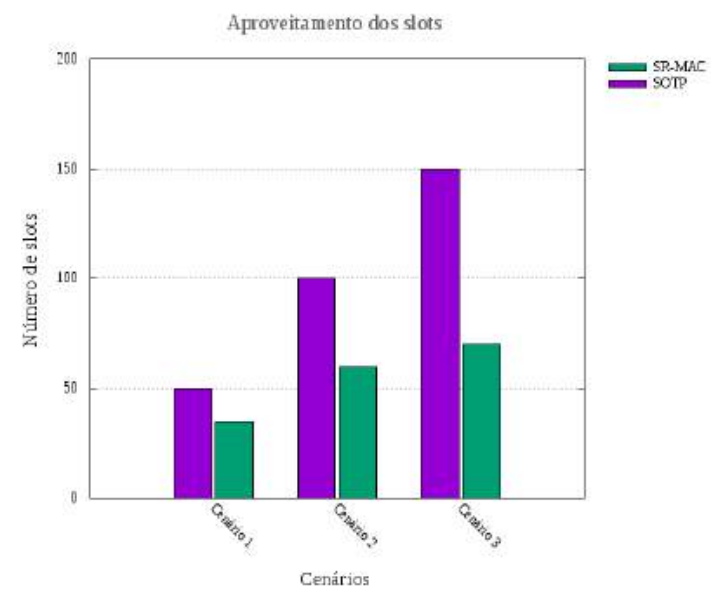

(a) Número de intervalos necessários.

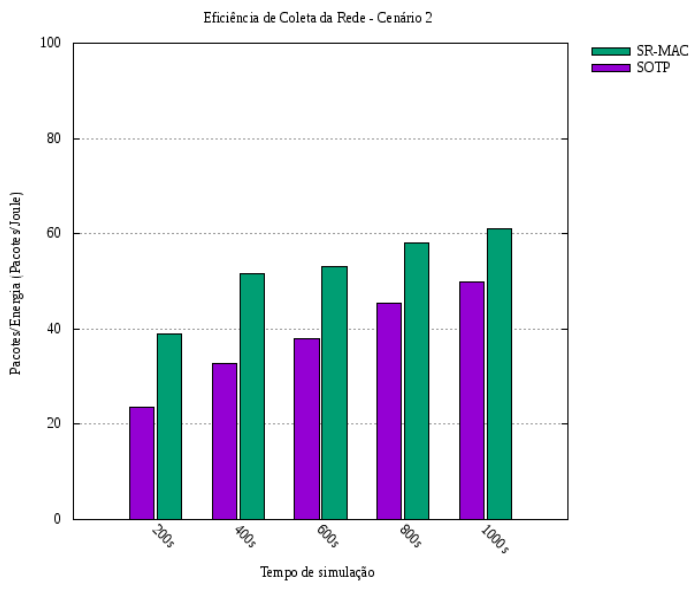

(c) Eficiência de Coleta no Cenário 2.

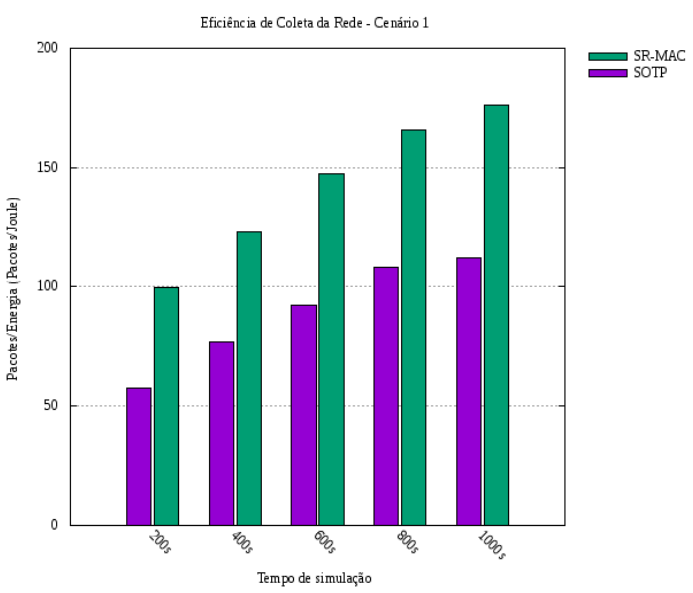

(b) Eficiência de Coleta no Cenário 1.

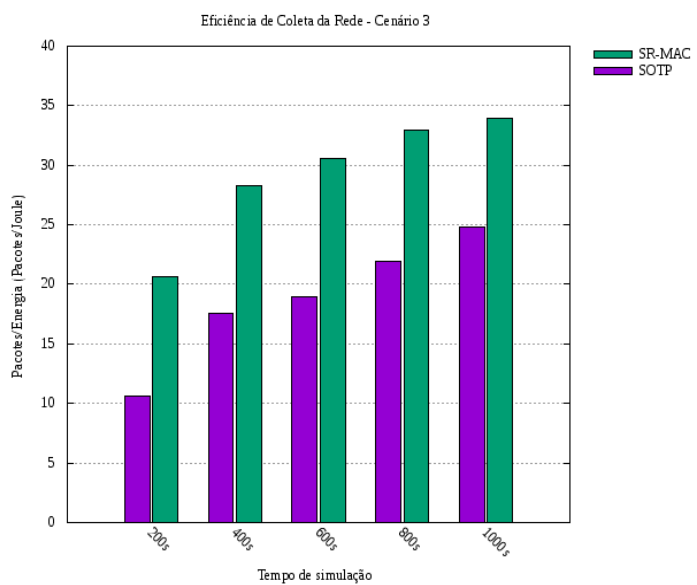

(d) Eficiência de Coleta no Cenário 3.

Figura 5. Métricas de Avaliação em relação à Estação Base.

A Figura 5(a) apresenta a quantidade de intervalos de transmissão necessários para cada cenário. Para estabelecer uma margem de segurança no SR-MAC em relação à quantidade de simulações realizadas e o número de intervalos necessários para cada cenário, esta métrica foi simulada 35 vezes e em cada simulação os nós foram depositados no ambiente de forma aleatória. Cada barra referente a cada cenário simulado representa a média das simulações realizadas. Observou-se que houve pouca dispersão dos dados em relação à média. O tempo de execução para estes testes foi de 200 s, pois foi observado que este era o tempo máximo para que todos os nós da rede se registrassem.

Nessa métrica, o protocolo SR-MAC, precisou de menos intervalos de tempo em comparação ao protocolo SOTP. Como não há reutilização no protocolo SOTP, a quantidade necessária de intervalos em cada cenário, equivale a quantidade de nós presentes na rede. O SR-MAC apresentou no cenário 1 uma taxa de eficiência entre 30-46\% em relação ao número de intervalos necessários em comparação ao SOTP. Já no cenários 2 e 3, o SR-MAC apresentou entre 40-50\% e 53,33\%-58\%, respectivamente. A tabela 2 exibe a margem de segurança para o protocolo SR-MAC em cada cenário. 
Tabela 2. Quantidade de intervalos necessários no SR-MAC.

\begin{tabular}{|c|c|}
\hline Cenário & Margem de Segurança \\
\hline 1 & 35 slots \\
\hline 2 & 60 slots \\
\hline 3 & 70 slots \\
\hline
\end{tabular}

As Figuras 5(b), 5(c) e 5(d) apresentam a eficiência de coleta através da quantidade de pacotes coletados pela estação base por energia gasta pela rede nos cenários 1, 2 e 3, respectivamente. Cada barra nos gráficos representa a média dos cálculos realizados para obter a eficiência de coleta em cada período das simulações realizadas. O cálculo demonstra a proporção entre o número de pacotes que chegam na estação base pela energia total gasta pela rede naquele período. Cada período estende-se de 0 até o valor descrito no eixo X de cada gráfico das Figuras 5(b), 5(c) e 5(d). A fórmula para o cálculo da eficiência de coleta em cada período segue abaixo:

$$
\frac{\operatorname{Pct} B S_{P}}{\sum_{i=1}^{n} E i_{P}}
$$

Onde $\operatorname{Pct} B S_{P}$ representa o número de pacotes recebidos pela estação base no período $P$, e $\sum_{i=1}^{n} E i_{P}$ o somatório de energia gasta por todos os nós da rede no período $P$. A partir dos resultados obtidos pelos gráficos acima, verifica-se que o protocolo SR-MAC apresentou uma eficiência de coleta maior que o protocolo SOTP. A eficiência de coleta pela estação base no protocolo SR-MAC é maior que no SOTP, como é possível ver na tabela 3, devido ao fato de que como o SR-MAC garante mais transmissões de dados, consequentemente, mais dados serão coletados mesmo que o consumo de energia da rede seja maior que o consumo no SOTP.

Tabela 3. Eficiência do SR-MAC em relação ao SOTP.

\begin{tabular}{|c|c|}
\hline Cenário & $1000 \mathrm{~s}$ \\
\hline 1 & $36,42 \%$ \\
\hline 2 & $19,28 \%$ \\
\hline 3 & $26,77 \%$ \\
\hline
\end{tabular}




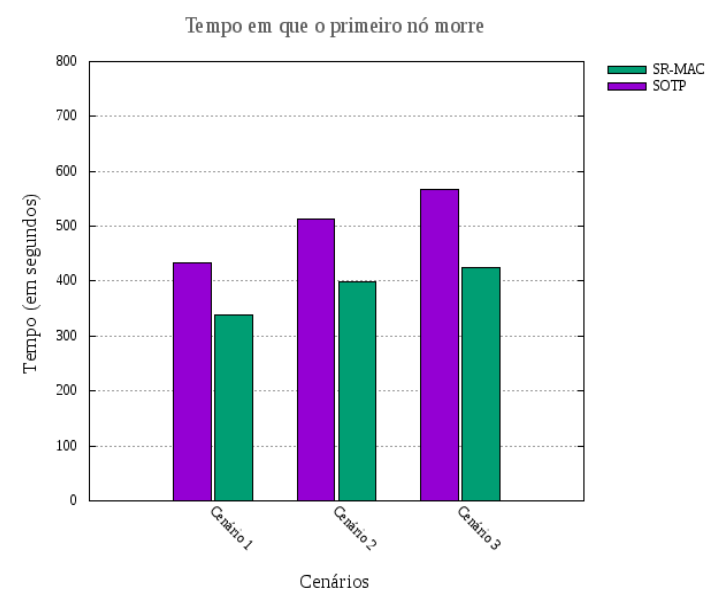

(a) Tempo de morte do primeiro nó.

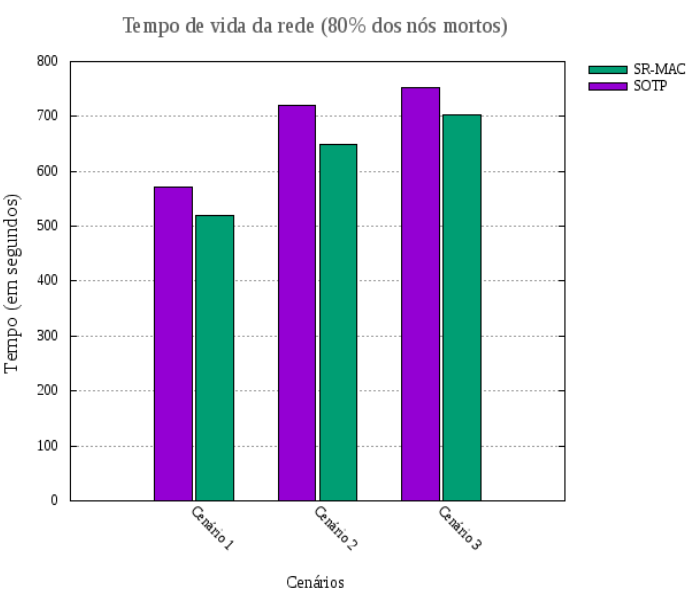

(b) Tempo de vida da rede.

Figura 6. Métricas de Avaliação em relação ao funcionamento da rede.

As Figuras 6(a) e 6(b) apresentam o tempo de morte do primeiro nó e o tempo de vida da rede com $80 \%$ dos nós mortos, respectivamente. Na Figura 6(b), cada barra colorida de cada cenário representa a média de tempo, dentre as 35 simulações realizadas, em que a rede continua a funcionar mesmo tendo atingido $80 \%$ de nós mortos. Para essas métricas, os resultados obtidos pelo protocolo SOTP foram melhores em comparação ao SR-MAC. No SR-MAC, os nós morrem cedo devido ao fato de que o quadro se repete mais vezes em comparação ao SOTP. Com isso os períodos de transmissão e recepção dos nós acontecem com mais frequência, ocasionando um maior consumo de energia. Já o quadro no SOTP, por ser maior, se repete com menos frequência, resultando em tempos maiores em modo de baixo consumo (sleep) e em tempos menores de transmissão e recepção. Em relação ao gráfico da Figura 6(b), o protocolo SR-MAC apresentou um percentual de somente $9 \%, 9,83 \%$ e $6,58 \%$ abaixo do tempo de vida do SOTP para o cenário 1,2 e 3 , respectivamente.

\section{Conclusão e Trabalhos Futuros}

Através dos resultados obtidos, foi possível avaliar o impacto dos novos mecanismos introduzidos no SR-MAC, que permitiram o reuso de intervalos TX e melhorias na eficiência da rede. Além disso, observou-se que o protocolo SR-MAC apresentou bons resultados tanto para uma rede menos densa (Cenário 1) como para uma mais densa (Cenário 3), proporcionando ganho nas métricas relacionadas ao aproveitamento dos slots (quantidade de intervalos necessários) e à eficiência de coleta da estação base em comparação ao SOTP. O protocolo proporciona escabilidade, possibilitando a inserção de mais nós na rede, gerando um aumento do fluxo de dados. O SR-MAC mostrou-se eficiente na coleta de dados captados pela estação base, mesmo os sensores apresentando um consumo de energia maior que os sensores no protocolo SOTP.

Para trabalhos futuros, pretende-se melhorar o SR-MAC em relação ao consumo de energia das baterias dos sensores e dar a possibilidade de recuperação da rede quando houver alguma falha na árvore de roteamento. Com isso, espera-se aumentar o tempo de vida da rede e tornar o protocolo ainda mais eficiente em relação ao fluxo de dados gerados pelos sensores e à coleta de dados captados pela estação base. Por fim, também pretende- 
se comparar o SR-MAC com outros protocolos existentes na literatura que tratam deste mesmo assunto e que apresentam características semelhantes ao protocolo SR-MAC.

\section{Referências}

Boulis, A. et al. (2011). Castalia: A simulator for wireless sensor networks and body area networks. NICTA: National ICT Australia.

Cunha, F. D., Cunha, Í., Loureiro, A. A., and Oliveira, L. B. (2013). Uma nova abordagem para acesso ao meio em redes de sensores sem fio. $31^{\circ}$ Simpósio Brasileiro de Redes de Computadores e Sistemas Distribuídos, pages 3-16.

El Gamal, A. and Kim, Y.-H. (2011). Network information theory. Cambridge university press.

Gajjar, S., Sarkar, M., and Dasgupta, K. (2014). Self organized, flexible, latency and energy efficient protocol for wireless sensor networks. International Journal of Wireless Information Networks, 21(4):290-305.

Goyal, D. and Tripathy, M. R. (2012). Routing protocols in wireless sensor networks: a survey. In 2012 Second International Conference on Advanced Computing \& Communication Technologies, pages 474-480. IEEE.

Huang, P., Xiao, L., Soltani, S., Mutka, M. W., and Xi, N. (2013). The evolution of mac protocols in wireless sensor networks: A survey. IEEE Communications Surveys \& Tutorials, 15(1):101-120.

Kurose, J. F. and Ross, K. W. (2010). Redes de Computadores e a Internet, volume $5^{\mathrm{a}}$ edição. Pearson.

Mitra, A. (2009). Lecture notes on mobile communication. A Curriculum Development Cell project Under QIP, IIT Guwahati, pages 157-166.

Rocha, L. S. (2012). Algorithmic aspects of graph colouring heuristics. PhD thesis, Nice.

Sgora, A., Vergados, D. J., and Vergados, D. D. (2015). A survey of tdma scheduling schemes in wireless multihop networks. ACM Computing Surveys (CSUR), 47(3):53.

Varga, A. (2014). Omnet++ user manual version 4.6. https : / / omnetpp. org/doc / omnetpp4/Manual .pdf. (Acessado em: 30/06/2015).

Wang, Y., Henning, I., Li, X., and Hunter, D. (2006). Sotp: a self-organized tdma protocol for wireless sensor networks. In Electrical and Computer Engineering, 2006. CCECE'06. Canadian Conference on, pages 1108-1111. IEEE.

Wu, D., Wang, G.-Y., and Li, X.-L. (2010). Distributed tdma scheduling protocol based on conflict-free for wireless sensor networks. In Intelligent Computing and Integrated Systems (ICISS), 2010 International Conference on, pages 876-879. IEEE. 